\title{
La reconnaissance des cultures indigènes
}

L'habitus missionnaire laïque de Pierre Deschamps (École normale supérieure de Saint-Cloud, 1892)

\section{Geneviève Zarate}

\section{(2) OpenEdition \\ Journals}

Édition électronique

URL : https://journals.openedition.org/dhfles/2952

DOI : $10.4000 /$ dhfles.2952

ISSN : 2221-4038

Éditeur

Société Internationale pour l'Histoire du Français Langue Étrangère ou Seconde

Édition imprimée

Date de publication : 1 décembre 2000

Pagination : 116-128

ISSN : 0992-7654

Référence électronique

Geneviève Zarate, "La reconnaissance des cultures indigènes », Documents pour l'histoire du français langue étrangère ou seconde [En ligne], 25 | 2000, mis en ligne le 20 janvier 2016, consulté le 08 décembre 2022. URL : http://journals.openedition.org/dhfles/2952 ; DOI : https://doi.org/10.4000/ dhfles.2952

Ce document a été généré automatiquement le 27 mai 2021.

Tous droits réservés 


\title{
La reconnaissance des cultures indigènes
}

\author{
L'habitus missionnaire laïque de Pierre Deschamps (École normale \\ supérieure de Saint-Cloud, 1892)
}

\section{Geneviève Zarate}

1 La figure du missionnaire marque en profondeur les instances qui ont accompagné, depuis plus de deux siècles, la diffusion de la langue française hors de France. Trois acteurs se partagent ainsi le statut de représentants des intérêts français dans le monde : le religieux, le militaire et l'enseignant. Chacun intervient à l'étranger avec les finalités et les moyens de sa propre légitimité, mais chacun est aussi le mandant d'une des composantes de la société française de la fin du XIX ${ }^{e}$ siècle. Les intérêts du religieux et du militaire sont étroitement associés quand leur solidarité est à la base de l'État français. Quand, au début du XXe siècle, en 1905, l'État se sépare des églises, les intérêts militaires et laïques fusionnent. Un nouvel idéal pédagogique est à construire, espace d'investissement des pionniers qui vont progressivement œuvrer à son institutionnalisation. Pierre Deschamps est l'une des figures de proue qui inaugure ce nouvel espace et occupe à l'heure actuelle une place de père fondateur.

2 À l'heure où l'École normale supérieure de Fontenay / Saint-Cloud opère une mue identitaire et devient l'ENS Lettres et sciences humaines en se délocalisant dans la région rhône-alpine, il n'est pas sans intérêt de faire resurgir le parcours pionnier de Pierre Deschamps, élève de l'ENS Saint-Cloud (promotion 1892). Au moment où se rapprochent les missions de l'Institut national de recherche pédagogique (INRP) et celles de la nouvelle ENS, il est de bon goût de penser, à moins de faire fi de l'histoire des idées scientifiques et de l'évolution des théories en sciences humaines, que les options à venir s'appuieront sur les bénéfices symboliques engrangés par l'École dans l'histoire de la diffusion du français hors de France et dans celle de la pensée pédagogique. Dans ce contexte a été menée une recherche intituléeTrajectoires familiales, scolaires et professionnelles des élèves de l'ENS de Fontenay / Saint-Cloud et relation à l'altérité. Le cas des promotions admises en 1986 et en 1996'. Ce portrait contribue à inscrire cette recherche dans une histoire institutionnelle. 


\section{Pierre Deschamps, fondateur de la Mission laïque}

Le travail de mémoire ébauché par la Mission laïque en 1982, et celui mené par l'Alliance française en 1983, permettent de reconstituer, pour une part, les conditions qui ont permis la fondation de ces institutions. Prenant acte de la malheureuse dissymétrie des sources ${ }^{1}$, on se propose de retracer le parcours militant de Pierre Deschamps, à l'origine de la création de la Mission laïque. Au début de sa carrière, il ne dispose pas des atouts dont sont ordinairement dotés les grands fonctionnaires de l'État. D'origine modeste, ce fils de garde- champêtre, né en province, dans l'Indre en 1873, intègre l'ENS de Saint-Cloud en 1892. À sa sortie, en 1894, on lui propose un poste de professeur à l'école normale de Tunis, au collège Alaoui, avec lequel l'ENS entretient déjà des relations privilégiées. Le choix d'un poste à l'étranger ne s'impose pas d'emblée à vingt ans : «J'accepte le poste de Tunis que vous m'avez fait proposer par M. Évrard, directeur de l'école normale de Châteauroux. Je l'avais refusé il y a deux mois, lorsque M. le Directeur de l'École de Saint-Cloud me l'avait offert. Je craignais alors de trouver de la part de mes parents une résistance que je n'aurais pu vaincre ». La famille de Deschamps a donc une perception dramatisée de l'expatriation. Il n'exerce à Tunis que brièvement pour prendre, en 1895 , le poste de directeur de l'école primaire centrale de Saint-Denis de la Réunion ${ }^{2}$. C'est là qu'il rencontre sa femme et se marie. Les documents, pourtant précis, sur lesquels nous nous appuyons ne permettent pas de savoir si cette dernière était d'origine réunionnaise ou française. Il prend, en 1898 et pour quatre ans, le poste d'inspecteur des écoles de l'Imerina,à Madagascaret, dès son arrivée, pour cause de défection du titulaire, celui de chef du service de l'enseignement à Madagascar jusqu'en 1902.

Du témoignage qu'il laisse de cette époque aux générations suivantes, il brosse de luimême le portrait d'un homme conscient de son inexpérience :

$\mathrm{Au}$ fait, j'étais comme si je venais de tomberà l'eau et je n'avais pas d'autre alternative que de me laisser coulerà pic et démissionner, ou essayer de surnager et de justifier l'espoir que, poliment, on semblait fonder sur moi, malgré ma jeunesse [...] je me rendais compte de mon isolement, de mon ignorance, et même, j'étais quelque peu inquiet de ne pas entrevoir, comme d'intuition, et ainsi que cela arrive parfois dans les situations difficiles, la voieà suivre ${ }^{3}$.

II n'y a guère de modèle sur lequel il puisse s'appuyer : « Il ne se trouva personne pour me tendre la perche : le Directeur de l'École le Myre de Vilers n'était pas beaucoup plus expérimenté que moi ». Les responsabilités sont importantes car tout est à faire : " Porté d'un seul coup à l'échelon supérieur du service, je n'avais pas d'autre chef que le général Galliéni : il m'assigna ma tâche sans tarder : contrôler les écoles des Missions et organiser l'enseignement officiel ».

Pierre Deschamps trouve les ressources nécessaires pour répondre à cette demande en puisant dans ses références de cloutier (les philosophes du XVIII ${ }^{e}$ ) et dans l'observation attentive du contexte malgache : «Évidemment, ce n'était pas en restant dans mon bureau [...] mais au contact des hommes et des choses du pays que je pouvais nourrir l'espoir de trouver une réponse, qui me satisfit, au moins à titre provisoire, au problème qui me tourmentait ". La solution vient en croisant deux cultures enseignantes opposées : d'une part celle des missionnaires religieux, pour lesquels le problème d'une contradiction culturelle ne se posait pas. "Ils étaient à Madagascar dans le but de « convertir » les Malgaches à la religion chrétienne [...] Les missionnaires 
peuvent donc puiser dans une expérience de quatre siècles [...] du moins n'ont-ils aucune hésitation sur le but à atteindre ". D'autre part sur une conviction intime : " construire, à côté de celui des Missions, un système positif d'évaluation propre à l'enseignement officiel, qui s'ajustât à la mentalité des Malgaches et ne valût que pour eux ». Deschamps cherche des réponses qui engagent la formation de l'individu tout entier, tant sur le plan intellectuel (" Comment l'âme malgache réagissait-elle à l'éducation qu'elle recevait des missionnaires catholiques ?») que professionnel (« L'École professionnelle ne tarda pas à révéler que [...] les Malgaches étaient aptes à tous les métiers ») et qui reposent sur une vision associative du partenariat colonial (« Il ne saurait être question, ce serait vain, de vouer les sociétés indigènes à l'immobilité ; ce qu'il faut, c'est les aider avec intelligence dans leur évolution »). La réponse est formulée en ces termes : "demander à la Société " laïque » de France un effort analogue à celui que faisaient les protestants et les catholiques pour la société des Missions évangéliques ».

De retour en France en 1902, Pierre Foncin (École normale supérieure d'Ulm), son aîné, impliqué dans la fondation de l'Alliance Française, lui accorde son appui pour fonder la Mission laïque. De 1909 à 1911, Pierre Deschamps prend un poste à Beyrouth. Il revient en France, supportant mal le séjour à l'étranger et souhaitant scolariser ses enfants en France. Il reprend en 1919 un poste pour un an à Beyrouth dans le but de réorganiser la Mission laïque au Liban après la guerre. Onze années d'expatriation marquent cette carrière qui s'achève en 1933 dans les fonctions de directeur de l'école primaire supérieure de Marseille. Un positionnement qui prend place dans une relation triangulaire qui soude objectivement hors de France l'idéal laïque avec les intérêts militaires de l'empire colonial, dans une opposition théorique avec les réseaux d'enseignement confessionnels, mais sur la base d'une alliance stratégique locale avec ceux-ci.

\section{L'engagement scout de Pierre Deschamps}

Dans cette quête de l'innovation où se joue l'avenir de la colonisation, le militant laïque engage sa vie professionnelle et sa vie privée. A son retour en France, cette expérience si particulière et pour laquelle il a renoncé à un parcours professionnel canonique, n'a pas de place objective dans le système éducatif hexagonal. Pierre Deschamps recycle son expérience internationale et son engagement militant en les mettant au service du mouvement scout à partir de 1920. On le retrouve, sous le nom de Zébu Jovial, sachem Éclaireur, pendant l'entre-deux-guerres, au Comité directeur des Éclaireurs de France de la région Provence ${ }^{4}$. Il s'intéresse surtout aux positions idéologiquement sensibles, « la propagande, les relations avec les comités et l'administration générale de la région ». Outre ses fonctions de Commissaire régional de Provence, il capitalise son expérience passée au Levant dans celles de Chargé des affaires pour la Syrie, qui se concrétisent par un camp organisé au Liban en août 1933. La même année, il inaugure le Foyer Scout International des Eclaireurs de France ${ }^{5}$. En cette fin de carrière professionnelle, l'expérience de l'étranger lui permet d'avoir une visibilité nationale dans le mouvement et d'être étroitement associé aux événements de portée internationale qui s'y déroulent : il accueille régulièrement Baden-Powell à Marseille en 1921, 1933, 1935, 1937. Ce réinvestissement militant hors du secteur scolaire proprement dit, à son retour de l'étranger, est un prolongement de l'esprit missionnaire. 
Cette deuxième vie de Pierre Deschamps n'est pas en soi si étonnante. Si le mouvement de Baden-Powell est d'obédience religieuse, celui des Éclaireurs cherche à s'en démarquer. Pierre Deschamps cherche à opérer, avec la référence scoute, la conversion qu'il a opérée au nom de l'école républicaine pour le bénéfice de la laïcité, non sans quelques acrobaties avec l'idée de neutralité :

Que nous donnions aux enfants, sur le vœu exprimé par leurs parents, toutes facilités pour qu'ils puissent pratiquer leur religion, c'est tout ce qu'on peut demander à notre neutralité. Mais, ces observations faites, et s'il est chimérique de concilier, quant aux principes, les conceptions du fondateur du scoutisme et celles de l'organisation de l'école laïque, reconnaissons qu'ils sont bien près l'un de l'autre, non seulement quand on les ramène sur le terrain solide de l'action, mais encore quand on considère la largeur de vues, l'élévation de pensée qui les caractérisent l'une et l'autre. Elles voisinent sur les hauteurs ${ }^{6}$.

Dans la volonté de concilier des positions intellectuelles qu'il avait à l'étranger vécues comme antagonistes, sans doute peut-on voir le désir d'un homme, rentré en France depuis 1907, devenu depuis 1920 cadre aux Éclaireurs de France, d'apporter à la société française une sensibilité qui ne peut être comprise que sous une formulation modérée. Il travaille ainsi pour un modèle de société, au plus large des structures éducatives classiques.

11 Mouvements scout et laïque, sont deux courants de pensée contemporains qui ont en commun des objectifs qui vont bien au-delà des pratiques scolaires et qui ont pour ambition de former des personnalités, de proposer une morale de vie. Tous deux s'inscrivent dans une perspective coloniale. Robert Stefenson Smyth Baden-Powell, général anglais, mena sa carrière militaire entre autres en Inde et en Afrique du Sud.Le Livre des éclaireuses comprend un chapitre intitulé « La vie à la frontière » qui débute en ces termes : " Les Éclaireuses doivent être préparées dans bien des [...] domaines. Il arrive à beaucoup de nos jeunes filles de devoir vivre dans les colonies. Bien souvent c'est une vie très dure, pleine d'aventures et d'imprévus ${ }^{7}$. Le manuel se présente comme une méthode permettant de développer le caractère et l'intelligence, l'adresse et l'habileté manuelles, l'esprit de service et de camaraderie, la santé physique et l'hygiène. Il emprunte à la culture militaire une conception rigoureuse de la hiérarchie (d'aspirante, l'apprentie passe au statut de lutin $2^{\mathrm{e}}$ classe puis $1^{\mathrm{e}}$ classe, d'éclaireuse $\left(2^{\mathrm{e}}\right.$ puis $1^{\mathrm{e}}$ classe), de guide puis de chef éclaireuse), du maintien corporel (l'ouvrage est ponctué du sigle TVD = Tenez-Vous Droite), la patrouille est l'unité de compte. Des épreuves permettent de passer d'un grade à un autre : le lutin $2^{\mathrm{e}}$ classe doit, par exemple, connaître l'histoire du drapeau britannique, savoir faire une série de nœuds et, version féminisée, savoir ourler un mouchoir ou un torchon à poussière... La référence aux anciens chevaliers se retrouve dans la " promesse de l'éclaireuse » qui, jurant de servir Dieu et le Roi, rejoint ainsi la tradition des cohortes de l'armée de Dieu. Cette idéologie patriotique se combine avec une éducationà la maîtrise de soi, avec le sens du devoir et du dévouement. L'instruction morale se traduit dans la pratique d'exercices physiques, dans la connaissance de la nature et des animaux, dans l'art de camper et dans les rudiments du secourisme. L'appel scout, petit journal ronéoté de Perpignan, présente ainsi le mouvement scout à ses nouveaux adhérents : " Le scoutisme est une éducation morale sous la forme plaisante d'un jeu - d'un grand jeu continuel - mais il exige l'obéissance à des principes librement consentis età une loi que l'on veut aimer, que l'on a fait sienne $»^{8}$. 


\section{Civilisation française et cultures indigènes : les positions d'un militantisme pédagogique laïque} françaises à l'étranger, les positions les plus avancées du prosélytisme laïque, particulièrement bien représentées à la Mission Laïque dans les années de fondation, ouvrent le débat sur les langues. Dans quelle langue l'enseignement doit-il être assuré ? En français, langue de la civilisation ? Dans les langues indigènes, langues vernaculaires? Pour le Congrès de la Mission laïque de 1906, le rapport Julien fait état de "l'enseignement des langues et des littérature indigènes dans les écoles indigènes des colonies ". La position de la Mission est d'autant plus constante sur ce point qu'elle est distincte de celle affichée par l'Alliance française : les langues indigènes doivent trouver une place dans la pédagogie coloniale.

Dans l'abstraction des intérêts immédiats, les arguments en faveur de l'emploi des langues indigènes pour la diffusion des contenus enseignés font valoir que "les indigènes possédant une forte culture en leur langue sont les mieux préparés pour en recevoir une autre en langue étrangère » et qu'il n'est pas légitime de "propager notre idiome parmi des populations de mœurs et de tournure d'esprit souvent bien différentes des nôtres ». Mais quand les finalités économiques sont mises en avant, il faut

...familiariser (les indigènes) avec notre idiome, non pour en faire de faux savants, pleins de morgue et de prétentions, mais d'utiles associés de nos colons, commerçants et industriels, des collaborateurs éclairés de nos Administrateurs, enfin, sous le même drapeau, des frères dévoués de nos soldats luttant ensemble pour la défense du patrimoine commun et d'un idéal devenu le même'.

L'apprentissage du français sert les intérêts économiques et militaires existant entre Français et indigènes. Les positions les plus ouvertes de la Mission sont en faveur de l'apprentissage des langues locales, requises dans la formation des enseignants coloniaux. Parmi les formules qui seront retenues pour l'enseignement aux indigènes, celle de l'enseignement professionnel sera prônée pour prendre en compte des réalités locales prosaïques et, plus stratégiquement, l'avenir et l'efficacité de la colonisation. Contrairement à l'opinion reçue, le ministère de l'Instruction publique est bien conscient de l'intérêt d'un enseignement adapté. Dès 1887, il envoie observer et faire un recensement systématique de ce qui se fait dans les pays du nord de l'Europe (Allemagne, Finlande, Suède, Norvège, Danemark), supposés plus avancés en ce qui concerne l'enseignement manuel et professionnel ${ }^{10}$. Pour concrétiser cette volonté de fonder un nouveau type d'enseignement, mise en pratique immédiate du mariage de la raison et du progrès dans l'éducation indigène, ce qui recouvre le nom de "pédagogie coloniale » prend corps à travers la création d'institutions. En 1902, ouvre l'École normale Jules Ferry, chargée de la formation des « instituteurs coloniaux », divisée dès 1904 en sections Afrique et Indochine. Si l'école ferme en 1913, sa création symbolise la prise de conscience de la complexité des réalités culturelles hors hexagone. Contrairement à ce que l'expression " enseignement professionnel " suggère, il s'agit moins d'apprendre un métier, objectif perçu comme trop exigeant et irréaliste pour les indigènes illettrés, que, sur le plan économique, de pourvoir en main d'œuvre qualifiée les colons et, sur le plan pédagogique, de faire raisonner l'enfant indigène sur des faits qui lui sont familiers en faisant passer au second plan des contenus d'enseignement 
centrés sur « les subtilités de la grammaire, les chinoiseries de l'orthographe, l'histoire chronologique des rois, les problèmes ou traquenards de robinets qui emplissent ou vident un bassin $»^{11}$. Sont estimés plus concrets l'enseignement manuel expérimental (par exemple, exercices de pliage, de tissage, de découpage et de cartonnage), et les leçons de choses agricoles, fondées sur des observations effectuées directement dans la nature. En Afrique occidentale française, sont inventées les écoles rurales, promues par le gouverneur général Brévié et poursuivies par Georges Hardy : chaque école devait avoir son « jardin scolaire ${ }^{12}$.

Ces positions représentent un front avancé de la pédagogie et sont soumises à la critique. Le débat de fond s'ordonne autour de la prise en compte des réalités culturelles locales dont la dimension confessionnelle ne représente qu'une facette. Les pédagogues militants impliqués dans l'enseignement aux colonies s'accordent sur l'influence politique que la France doit exercer dans ses colonies, sur le rôle majeur que la civilisation, entendue au sens laïque, doit jouer dans l'éducation :

... honneur, loyauté, justice, respect des faibles, respect des traités, droits de l'homme et droits des peuples. C'est sur ce terrain où se rencontrent les hommes civilisés que nous voulons édifier notre œuvre d'éducation. «Civiliser ", voilà notre programme $»^{13}$.

Une ligne idéologique sépare les partisans de l'assimilation culturelle et les partisans d'un enseignement prenant étroitement en compte la culture, la langue et les modes de vie et de pensée locaux. Les premiers justifient leurs positions en ces termes :

Dans la plupart des colonies, et sauf exception pour les « vieilles " colonies où l'assimilation a accompli son œuvre depuis longtemps, la vie politique et sociale des indigènes n'a pas été sensiblement modifiée par la domination française ; [...] mais cette vie politique et sociale est plus régulière que par le passé, mieux garantie contre les abus de pouvoir et les exactions ${ }^{14}$.

Les seconds, soucieux de promouvoir le développement indigène, voient leurs choix illustrés par cette métaphore : «Parlant de l'âme malgache, je l'ai souvent comparée à une humble fleur sauvage qui n'a pas l'orgueilleuse beauté des fleurs de nos jardins, mais qui n'en est pas moins agréable à connaître $»^{15}$. Quelles que soient les positions rapprochées de ces discours militants, renseignement laïque, engagé dans une action qui doit être exemplaire face aux méthodes traditionnelles de l'enseignement confessionnel, est devant une obligation de qualité qui mobilise énergies et dévouement.

\section{La référence missionnaire laïcisée et la formation d'enseignants}

Pour faire face à la concurrence des missions religieuses qui ont le privilège des positions historiquement établies et le handicap des valeurs conservatrices, le missionnaire laïque est un acteur au service du progrès et de la modernité. L'éducation des indigènes est un pari qui, pour réussir, mérite des réponses originales face à des besoins spécifiques. De nouveaux modèles sont à inventer. Deschamps invente le stage à l'étranger pour les instituteurs nouvellement recrutés à Madagascar :

Tananarive devint un poste de début ; par mes soins, il n'y eut nulle part ailleurs de poste vacant à attribuer aux nouveaux instituteurs arrivant de France ; le séjour à Tananarive prit le caractère d'un stage [...] Le premier conseil que je lui donnais 
était de se garder de juger les Malgaches avec prévention, par comparaison avec lui-même, mais de les observer, d'essayer de les comprendre ${ }^{16}$. rabais. Il fait l'objet au contraire de propositions qui s'affichent clairement comme novatrices pour relever le double enjeu d'un enseignement par lequel le prestige de la France doit s'affirmer, et qui doit répondre à une demande qui promet d'être massive. Si on reconduit des méthodes existantes dans le système français, par exemple l'explication des textes de lecture, c'est en se posant systématiquement la question de leur adaptation à l'étranger. La leçon d'histoire où Bakoto, élève malgache, se donne les Gaulois pour ancêtres est un objet d'effroi pour Deschamps en tournée d'inspection à Madagascar. On propose ainsi à la Mission laïque des structures éducatives qui visent à restituer le contexte étranger, comme la notion de "musée scolaire ». L'ambition messianique se trouve bien servie par les progrès technologiques et la Méthode Weill intitulée Leçons de français est préconisée dans le Bulletin de la Mission Laïque Française pour son utilisation du phonographe et ses disques de la Maison Pathé, permettant d'acquérir " une prononciation parfaite $»^{18}$. Les prémices d'une analyse des besoins se dessinent dans le questionnaire mis au point par le docteur Papillault. Il cherche à mettre en évidence les particularités du public de la Mission laïque française, à dégager

...les aptitudes particulières des enfants que la Mission laïque a entrepris d'éduquer,

à marquer les caractères distinctifs qu'ils présentent avec les enfants de France, et à

fournir ainsi tous les éléments pour une bonne orientation de l'enseignement, adapté d'une façon précise aux tendances mentales de chaque population ${ }^{19}$.

Il s'agit d'apporter des réponses rationnelles, "scientifiques ", selon l'expression du docteur Papillault, à la diversité culturelle à laquelle les enseignants sont confrontés. Le souci de fournir des réponses pédagogiques spécifiques est bien là. Que l'idée de progrès technique soit associée à l'expansionnisme des intérêts français dans le monde, cela n'a rien d'étonnant : la France doit montrer le meilleur d'elle-même quand il s'agit d'affirmer sa prééminence. Mais le meilleur produit d'exportation reste sans aucun doute, aux yeux des contemporains, la foi que la France accorde dans son système d'enseignement laïc, symbole de sa civilisation. La position de la Mission laïque se démarque des positions étroitement patriotiques, en plaidant pour une reconnaissance des cultures indigènes, reconnaissance qui constitue un préalable indispensable à l'efficacité de la mission colonisatrice. Cette position est celle des progressistes de l'époque.

L'enseignement aux colonies se doit d'être « scientifique et rationnel », selon l'article 3 des statuts de la Mission laïque. Quand le débat national doit s'appliquer à la notion de race, se posent des questions inédites au regard de la pédagogie hexagonale ${ }^{20}$. À la Mission laïque, deux discours vont se trouver systématiquement juxtaposés : au nom du progrès, on peut justifier la ségrégation entre enfants métis et enfants français :

Même avec l'application restrictive du droit de reconnaissance, lesSociétés ditesdes enfants métis en favorisant et en consacrant la formation d'une classe sociale intermédiaire entre les Français et les indigènes, présentent un danger pour 
l'avenir de la colonisation, alors que l'introduction dans la race indigène d'éléments nouveaux plus intelligents constitue pour celle-ci une amélioration progressive conforme aux lois de l'évolution ${ }^{21}$.

23 Par ailleurs, au nom du rationalisme, il convient de définir une politique œcuménique vis-à-vis de la civilisation française et de celle des indigènes. La visée assimilationniste est la position tenue par les partisans d'une définition dure du patriotisme, la voie de l'association est celle du réalisme : «Faut-il, de ce que nous venons de dire, conclure (l'assimilation étant irréalisable) que les puissances coloniales devraient renoncer à toute entreprise d'expansion extérieure ? Telle n'est pas notre pensée car ce serait renier ici l'œuvre magnifique de la Troisième République $»^{22}$. Le modèle associationniste, " heureuse formule ", est donc le fait d'un compromis qu'impose l'irréductibilité des races. Le partage d'un idéal commun est la finalité éducative de l'enseignement du français : « Nous percevons bien que d'un mot l'on nous marque le but à atteindre $:$ il faut les civiliser $» .^{23}$

\section{Un profil professionnel missionnaire sécularisé}

Les parcours fondateurs sont exemplaires dans la mesure où ils empruntent au modèle confessionnel un esprit missionnaire, tout en le réaménageant au nom d'un idéal humaniste et patriotique.

Un missionnaire doit être un excellent voyageur. Obligé de parler la langue des peuples auxquels il prêche l'Évangile, de se former à leurs usages, de vivre longtemps avec toutes les classes de la société, de chercher à pénétrer dans les palais et dans les chaumières, n'eût-il reçu de la nature aucun génie, il parviendrait encore à recueillir une multitude de faits précieux ${ }^{24}$.

Ce portrait brossé par Chateaubriand, rendant hommage à la présence jésuite dans le monde, est fidèle au modèle, cette fois sécularisé, prôné par Deschamps. Ce modèle évolue au cours du siècle suivant en accentuant la dimension anticléricale, en se centrant sur une pédagogie novatrice, en oeuvrant pour un militantisme qui célèbre l'esprit d'entreprise et qui se soucie de la concurrence internationale imposée par l'hégémonie progressive de la langue anglaise. Un siècle plus tard, un ensemble de propriétés sociales communes caractérisent les acteurs de la diffusion du français. Celles-ci se déclinent dans un espace qui est d'autant plus géographiquement ouvert qu'il est dépendant des valeurs nationales. Qu'ils soient hommes ou femmes expatriés, exerçant dans un lycée français à l'étranger et garants d'une conformité exacte par rapport au modèle national ou affectés dans une Alliance française ou dans un centre culturel français et soumis aux règles drastiques de la clientèle, les enseignants français expatriés déclinent, tout au long de la première moitié du $\mathrm{XX}^{\mathrm{e}}$ siècle, les différentes formes d'un habitus missionnaire qui a oublié, en les incorporant sous une forme indiscutée, les propriétés socialement exigées au moment de la création de cet espace professionnel.

Enseigner à l'étranger dans ces années-là, c'est vivre une aventure hors du commun et mettre le savoir au service de l'humanité. Appelée bien plus tard assistance, coopération ou action humanitaire, l'aide à l'étranger fait découvrir à ceux qui se mobilisent pour ses objectifs une réalité de «terrain », faite d'épreuves, inconnues dans le pays où l'enseignant a été socialisé, d'un ailleurs aux mœurs déroutantes qu'il faut apprivoiser, et de nouvelles hiérarchies culturelles et administratives dans lesquelles il faut s'inscrire. Ingéniosité, débrouillardise, esprit pratique sont les qualités exigées de 
l'enseignant français expatrié pour triompher de l'inattendu. Pour la cause, des tâches, qui seraient inacceptées dans le profil un poste classique, deviennent une nécessité indiscutée, le signe d'une liberté et d'une autonomie impensables en métropole. L'idéal scout est une autre matrice qui dicte les comportements valorisés dans la sélection des enseignants exerçant hors de France. Les positions de Pierre Deschamps solidarisent cet idéal scout avec la culture normalienne et préfigurent un modèle d'engagement éducatif à l'étranger qui sera largement valorisé pendant le $\mathrm{XX}^{\mathrm{e}}$ siècle.

Le projet militant de l'enseignement hors de France repose sur une vision où l'espace étranger n'est pas le simple prolongement de celui de la métropole. Aux yeux de la politique intérieure française, l'étranger est un enjeu de prestige international qui impose une exigence de réussite et qui suppose une prise de risque : la France y joue son rapport privilégié avec l'Universel. Progressivement, au nom des intérêts de l'État français, l'enseignement qui se fait hors de l'hexagone se dote d'un espace d'intelligibilité propre, avec ses réseaux, ses principes d'action et de perception du monde, ses modes de catégorisation qui progressivement imposent leur radicale différence. Serviteurs de la pensée laïque et des intérêts les plus puissants de l'État français, les acteurs de cet espace en expansion peuvent développer une position paradoxale : ils sont les représentants de l'État mais peuvent en inverser les valeurs sans nuire à ses intérêts fondamentaux. Au moment où se séparent les églises et l'État, l'Alliance française peut revendiquer une position œcuménique, associant dans sa stratégie de conquête, valeurs laïques et réseau d'influence confessionnels. Alors que se joue une relation exclusive à la civilisation, le militant laïque peut plaider la prise en compte des réalités locales et l'aménagement de la différence culturelle.

En ce début de siècle, après la dissolution du Centre de Recherche et de Formation en Éducation (CREFED) et du Centre de Recherche et d'Étude pour la Diffusion du Français (CREDIF) en 1996, et la nécessité de présenter une politique non seulement européenne, mais internationale, que fera l'ENS Lettres de la région Rhône-Alpes de son histoire et de celle de militants qui ont donné naissance à une institution toujours en activité et qui ont donné corps à des positions d'avant-garde dans le domaine de la pédagogie visà-vis de l'étranger?

\section{NOTES}

1. On peut estimer que les travaux sur l'Alliance française sont encore insuffisants au regard du rôle et de l'importance de cette institution. En dépit des apports de l'article d'Ivan Barko icimême et de la thèse de Claude Cordier intitulée Institution de l'Alliance française et émergence de la francophonie. Politiques linguistiques et éducatives (1880-1914), Université Lumière Lyon II, 1997.

2. Burrows M. « La fondation et les premières années de la Mission laïque » in :Éléments pour une histoire, pp. 56-60.

3. Deschamps P. «Promenade archéologique (à travers les « souvenirs de ma vie coloniale que je raconte à mes enfants et petits enfants ») » in :Bulletin de Saint-Cloud $n^{\circ} 1$, février 1953. Cité in :Éléments pour une histoire (1982) p. 82. 
4. Palluau N. Permanence et évolution d'un mouvement du scoutisme des années 30 à la Libération à travers l'exemple des Éclaireurs de France en Vaucluse. Mémoire de maîtrise. Aix en Provence : Université de Provence, 1992.

5. Bulletin des Collines Ensoleillées, nos 148-149-150, mai-juin-juillet 1933, in Palluau, 1992, p. 43.

6. Deschamps P. cité par Kergomard P., François P. Histoire des Éclaireurs de France de 1911 à 1951. Paris : Eclaireurs et éclaireuses de France ? 1983, p. 69.

7. Baden-Powell Le livre des éclaireuses. Manuel pour les lutins, les éclaireuses, les guides et leur chef. Paris : Neuchâtel, Delachaux et Niestlé. (Collection actualités pédagogiques). Traduction de l'anglais K. Jentzer. Titre anglais Girl Guiding.

8. L'appel scout, $n^{\circ} 4$. Perpignan, juin 1930.

9. Rapport Julien «Enseignement des langues et des littératures indigènes dans les écoles indigènes des colonies »in " Congrès de la Mission de 1906 », cité dansÉléments pour une histoire (1982), p. 125.

10. Salicis G. A. « De l'enseignement manuel et professionnel en Allemagne et dans les pays du nord. Rapport à M. le ministre de l'Instruction publique sur une mission relative à l'enseignement du travail manuel dans divers pays étrangers ».Mémoires et documents publiés par le Musée pédagogique. №14. Paris : Delagrave, 1887.

11. Rapport Rocheron sur l'éducation professionnelle. Congrès 1906. «L'association : une chance pour les colonisés. Un enseignement adapté à leurs besoins, facteur de développement ». Reproduit in :Éléments pour une histoire (1982), p.133-138.

12. Bouche D. «Une expérience oubliée : l'école rurale en Afrique occidentale française dans la première moitié du XX $\mathrm{X}^{\mathrm{e}}$ siècle » in : Documents pour /' histoire du français langue étrangère ou seconde. Paris : SIHFLES, $n^{\circ} 7$, juin 1991. p. 18-24.

13. Besnard E. « La France en Orient ». Conférence, inBulletin de la Mission Läque Française, $\mathrm{n}^{\circ} 3$, 1918, in : Éléments pour une histoire (1982), p. 200.

14. Hardy G. (1943), Histoire de la colonisation française, Larose, p. 227.

15. Deschamps P. (1953). Cité dans : Éléments pour une histoire (1982) p. 88.

16. Deschamp P., «Promenade archéologique (à travers les souvenirs de ma vie colonoale que je raconte à mes enfants et petits enfants)» in Bulletin de St-Cloud, $n^{\circ} 1$, février 1953. Cité dans : Éléments pour une histoire (1982), p. 94.

17. Buisson F., Manuel général. Mai 1905.

18. Bulletin de la Mision Laïque Française, $n^{\circ} 1$, juin 1918. Reproduit dans Éléments pour une histoire (1982), p. 209.

19. $3^{\mathrm{e}}$ congrès de laMission Laïque Française. Bulletin de la Mission Lä̈que, 1913. Reproduitin Éléments pour une histoire(1982), p. 154.

20. Lors d'un passage à Saint-Cloud, P. Deschamps se fait remettre L'Esprit nouveau d'Edgard Quinet par son ancien professeur de philosophie.

21. Congrès de 1906, cité dans Éléments pour une histoire (1982), p. 114.

22. Rapport Julien, op. cit., p. 125

23. Congrès de 1906 de la Mission laïque. «Rapport concernant le rôle et la préparation des instituteurs coloniaux », in Eléments pour une histoire (1982), p. 139.

24. Chateaubriand F. R.,Le Génie du Christianisme, 1802. Quatrième partie, Livre 4, Chapitre 9. Fin des missions. 
INDEX

Mots-clés : culture indigène, civilisation française, mission laïque, Pierre Deschamps, colonisation

Keywords : Native culture, French civilization, Pierre Deschamps, colonization

\section{AUTEUR}

\section{GENEVIÈVE ZARATE}

École normale supérieure de Fontenay / St Cloud et Université de Paris III 